\title{
Repurposing drug molecule against SARS-Cov-2 (COVID-19) through molecular docking and dynamics: a quick approach to pick FDA-approved drugs
}

\author{
Nabeela Farhat ${ }^{1} \cdot$ Asad U. Khan ${ }^{1}$ \\ Received: 19 September 2020 / Accepted: 17 September 2021 / Published online: 2 October 2021 \\ (c) The Author(s), under exclusive licence to Springer-Verlag GmbH Germany, part of Springer Nature 2021
}

\begin{abstract}
A novel coronavirus known as severe acute respiratory syndrome is rapidly spreading worldwide. The international health authorities are putting all their efforts on quick diagnosis and placing the patients in quarantine. Although different vaccines have come for quick use as prophylactics, drug repurposing seems to be of paramount importance because of inefficient therapeutic options and clinical trial limitations. Here, we used structure-based drug designing approach to find and check the efficacy of the possible drug that can inhibit coronavirus main protease which is involved in polypeptide processing to functional protein. We performed virtual screening, molecular docking and molecular dynamics simulations of the FDAapproved drugs against the main protease of SARS-CoV-2. Using well-defined computational methods, we identified amprenavir, cefoperazone, riboflavin, diosmin, nadide and troxerutin approved for human therapeutic uses, as COVID-19 main protease inhibitors. These drugs bind to the SARS-CoV-2 main protease conserved residues of substrate-binding pocket and formed a remarkable number of non-covalent interactions. We have found diosmin as an inhibitor which binds covalently to the COVID-19 main protease. This study provides enough evidences for therapeutic use of these drugs in controlling COVID-19 after experimental validation and clinical demonstration.
\end{abstract}

Keywords Coronavirus · Inhibitors · Drug repurposing $\cdot$ Protease

\section{Introduction}

A novel coronavirus termed as severe acute respiratory syndrome coronavirus 2 (SARS-CoV-2) is responsible for the respiratory disease in China. The SARS-CoV-2 outbreak was declared as an international concern of public health emergency by the World Health Organization [1-3]. Most of the infected patients have mild symptoms such as fever and cough, but it can be fatal leading to pneumonia and acute respiratory failure, particularly in older males with comorbidities [4]. Currently, any specific therapeutic alternatives for the infection is not present, and the treatment depends on the symptom along with repurposing of antiviral drugs such as ritonavir along with antibiotics for treatment

Asad U. Khan

asad.k@rediffmail.com

1 Medical Microbiology and Molecular Biology Laboratory, Interdisciplinary Biotechnology Unit, Aligarh Muslim University, UP 202002 Aligarh, India of secondary infections [1] The obstruction of proteolytic processing of polyproteins by inhibiting necessary viral proteases has been a fruitful strategy in the treatment of human immunodeficiency virus (HIV) and hepatitis C $[5,6]$. This proves that treatment of viral infections by inhibiting the protease will be a successful approach, since the main protease of SARS-CoV-2 is assumed to play an essential role in viral replication, therefore considered as a promising target $[7,8]$. The main protease is composed of 306 amino acids with enzymatic activity for replication in coronavirus. It also plays a significant role in processing of the polypeptide into functional proteins [9]. The SARS-CoV-2 main protease crystal structure was recently solved it is a homodimer (designated protomer A and B). Each protomer is composed of three domains: domain I (residues 8-101) and domain II (residues 102-184) comprises an antiparallel $\beta$-barrel structure, while domain III (residues 201-303) has five $\alpha$-helices arranged into a largely antiparallel globular cluster, and it is connected to domain II by a long loop region (residues 185-200). The substrate-binding site in SARS-CoV-2 Mpro has a Cys-His catalytic dyad and is located in a cleft between 
domain I and domain II [10], which enables the designing of inhibitory compounds. SARS-CoV-2 and SARS-CoV-1 proteases' sequence similarity is $96.1 \%$ [7, 11], so SARS-CoV-2 might be treated with the drugs developed against SARSCoV-1. [12]. But these compounds are not the approved medicine [13]. Moreover, the differences in single amino acids might affect its effectiveness for the novel virus [14]. Hence, SARS-CoV-2 main protease inhibitor development is an urgent need. Crystal structure analysis proposes that the residues in the active site of SARS-CoV-2 Mpro are Thr25, Thr26, Leu27, His41, Ser46, Met49, Tyr54, Phe140, Leu141, Asn142, Gly143, Cys145, His163, Met165, Glu166, Leu167, Pro168, Phe185, Asp187, Gln189, Thr190, Ala191 and Gln192. These residues play an important role in the development of effective inhibitors against SARS-CoV-2 main protease [15]. Various studies have reported different inhibitors like ligand N3 [10], compounds 11a and 11b [16] and taxifolin [17]. However, all these compounds have to pass several phases before clinical use. So FDA-approved drugs are great choice. In this study we have screened FDAapproved drug library against the main protease of SARSCoV-2 and further validated the selected compounds by computational method.

\section{Result and discussion}

\section{Virtual screening against Covid-19 protease}

The virtual screening is one of the most frequently used techniques which helps to minimize the time and cost [18, 19]. Screening of the potentially active selected drug against biological targets was carried out using GOLD software. Among 6054 compounds screened from superdrug2 database, six have been selected for further validation. These six molecules are selected on the basis of gold score and number of hydrogen bonds. The highest GOLD fitness score among the selected compound was found 79.7 for amprenavir, whereas other compounds have GOLD fitness of 70.6 (cefoperazone), 64.52 (Riboflavin), 64.25 (Diosmin), 63.57 (Nadide) and 61.39 (Troxerutin) as shown in Table 1.

\section{Binding modes generated by molecular docking}

Binding modes of protein and ligands were studied by measuring the binding energy obtained from docking tool AutoDock Vina and inspection of physical and chemical bonding between protein and drug molecules. Docking scores of all proteins are shown in Table 1. Different types of interactions namely hydrophobic, hydrogen bonding and other interactions were monitored during protein-ligand complexes which are listed in Table 1. The hydrogen bonds with taxifolin were formed by residues Glu166, Thr190, Tyr54,

Table 1 Ligand structure, gold score, AutoDock Vina binding energy and amino acid involved in various type of interaction

\begin{tabular}{|c|c|c|c|c|c|c|}
\hline Inhibitors & Gold Score & $\begin{array}{l}\text { Auto- } \\
\text { Dock } \\
\text { energies } \\
\text { (kcal/ } \\
\text { mol) }\end{array}$ & Hydrogen bonds & $\begin{array}{l}\text { Hydrophobic } \\
\text { bond }\end{array}$ & Other & $\begin{array}{l}\text { Binding energy } \\
\text { by g_mmpbsa }(\mathrm{KJ} / \\
\mathrm{mol})\end{array}$ \\
\hline Chloroquine & 58 & -5.1 & $\operatorname{Arg} 188$, Gln189 & - & $\begin{array}{l}\text { Met165 } \\
\text { (Pi-Sulphur) }\end{array}$ & $-170+/-37$ \\
\hline Taxifolin & 57 & -7.5 & $\begin{array}{l}\text { Glu166, Thr190, Tyr54 } \\
\text { Asp187, Pro168 }\end{array}$ & Pro168, Met165,His41 & $\begin{array}{l}\text { Met165 } \\
\text { (Pi-Sulphur) }\end{array}$ & $-276+/-32$ \\
\hline Amprenavir & 79.7 & -8 & $\begin{array}{l}\text { Gly143, Thr24, } \\
\text { Cys145, } \\
\text { His164 }\end{array}$ & - & - & $-341+/-48$ \\
\hline Cefoperazone & 70.6 & -8.4 & $\begin{array}{l}\text { Thr45, Thr24, } \\
\text { Ser46, Glu166 }\end{array}$ & - & - & $-398+/-69$ \\
\hline Nadide & 63.57 & -9.1 & $\begin{array}{l}\text { Asn142, Cys145, } \\
\text { Leu141,Glu189 }\end{array}$ & - & - & $-154+/-25$ \\
\hline Diosmin & 64.25 & -8.2 & $\begin{array}{l}\text { Arg188, Thr 26, Gln189, Asn142, } \\
\text { Cys } 145\end{array}$ & - & $\begin{array}{l}\text { Met165 } \\
\text { (Pi-Sulphur) }\end{array}$ & $-282+/-40$ \\
\hline Riboflavin & 64.52 & -8 & $\begin{array}{l}\text { Gly143, Asn142, } \\
\text { Ser144, His163, } \\
\text { Cys145 }\end{array}$ & His41,Met49 & $\begin{array}{l}\text { Met165 } \\
\text { (Pi-Sulphur) }\end{array}$ & $-287+/-63$ \\
\hline Troxerutin & 61.39 & -8.2 & $\begin{array}{l}\text { Gly143, Cys145, His163, Leu141, Ser144, } \\
\text { Thr190 }\end{array}$ & His41, Met165 & - & $-357+/-57$ \\
\hline
\end{tabular}


Asp187 and Pro168. Notably, in all possible inhibitors (except cefoperazone), Cys145 and oxyanion hole residues Asn142 and Gly143 were playing an important role in stabilising complex by forming hydrogen bond. Gly143 stabilised the complex of protease-amprenavir, protease- riboflavin and protease-troxerutin, while Asn142 played role in stability of protease-nadide complex, protease-diosmin complex and protease-riboflavin. However, in protease-cefoperazone complex, Thr45, Ser46 and Glu166 were found to have a crucial role in stability. The protease-nadide complex is additionally stabilised by Leu141 and Glu189. In addition, residues Thr26, Gln189 and Met165 are playing significant role in stability of protease-diosmin complex. Furthermore, in protease- riboflavin, His163, His41, Met49 and Met165 stabilise the complex. Moreover, His163, Leu141, Thr190, His41 and Met 165 have crucial role in stability of proteasetroxerutin complex.

\section{Binding modes refined by MD simulation}

MD simulation is extensively applied to monitor the stability of protein-drug/protein/DNA complexes, to restore the binding energy of protein complexes and to study the conformational changes occurring during ligand binding and unbinding, protein folding and protein dynamics. Here MD simulation was executed for all protein ligand complexes to refine and check the binding modes obtained by molecular docking through AutoDock Vina. The 2D structure of all the ligands are shown in Fig. 1.

Key determinants for drug specificity are hydrogen bonds [20], and all inhibitors have an optimal number of average hydrogen bonds as taxifolin, which proved them as good inhibitor molecules. Higher average number of hydrogen bonds in diosmin, nadide and troxerutin were observed in comparison to taxifolin and chloroquine (Fig. 2).

The protein structure conformational stability during the course of the simulation was determined by the RMSD. The predicted docked complexes RMSD analysis showed stable behaviour, confirming the strong binding affinity and the interaction between docked complexes. All the inhibitorbound complexes attained equilibrium during $100 \mathrm{~ns}$ trajectory which was evident with RMSD plot. RMSD graph was stable for cefoperazone, diosmin, riboflavin, taxifolin, chloroquine and troxerutin, while amprenavir and nadide have shown slight deviation (Fig. 3). A minimum RMSD of backbone atoms strongly suggests stable dynamic behaviour of docked complex. It was clear that trajectories of all docked complexes remained stable with proper conformations throughout all the three replica of $100 \mathrm{~ns}$, indicating that ligands were stable at the active site of protein during interactions (Fig. 3 and S1).

Compactness and shape of protease inhibitor complexes were observed by measuring the $\mathrm{Rg}$ value. If protein unfolds, Rg change with time, while a stably folded protein maintains a steady value of $\mathrm{Rg}$. Nadide seems to form the most stable

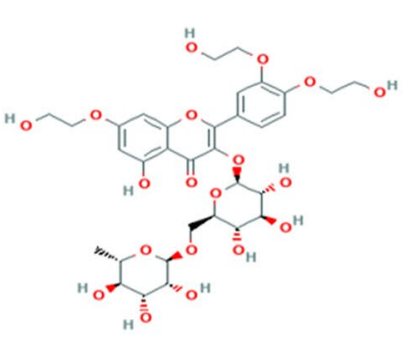

(a)

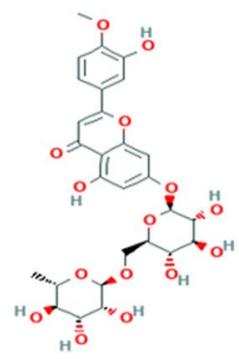

(e)

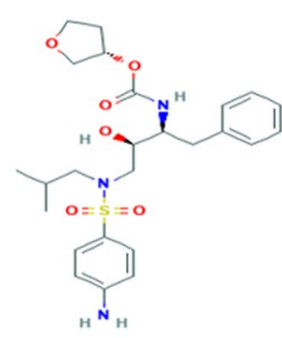

(b)

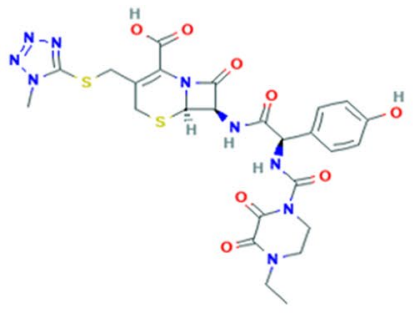

(c)

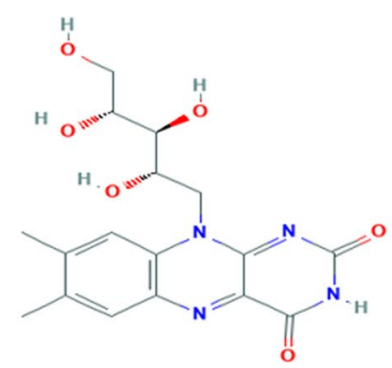

(g)

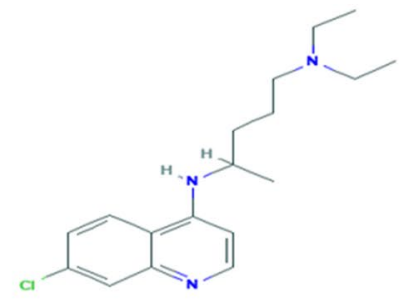

(d)

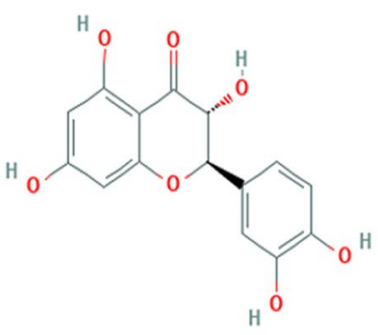

(h)

Fig. 1. 2D diagram of ligand structure: (a) troxerutin, (b) amprenavir, (c) cefperazone, (d) chloroquine, (e) diosmin, (f) nadide, (g) riboflavin, and (h) taxifolin 
Fig.2 Binding site active site amino acid involved in binding and hydrogen bond analysis of protein-ligand complex, (a) protease-taxifolin complex, (b) protease-riboflavin complex, $(\mathbf{c})$ protease-amprenavir complex, (d) protease- nadide complex, (e) protease-troxerutin complex, (f) protease-diosmin complex, (g) protease-cefoperazone complex and (h) protease-chloroquine complex
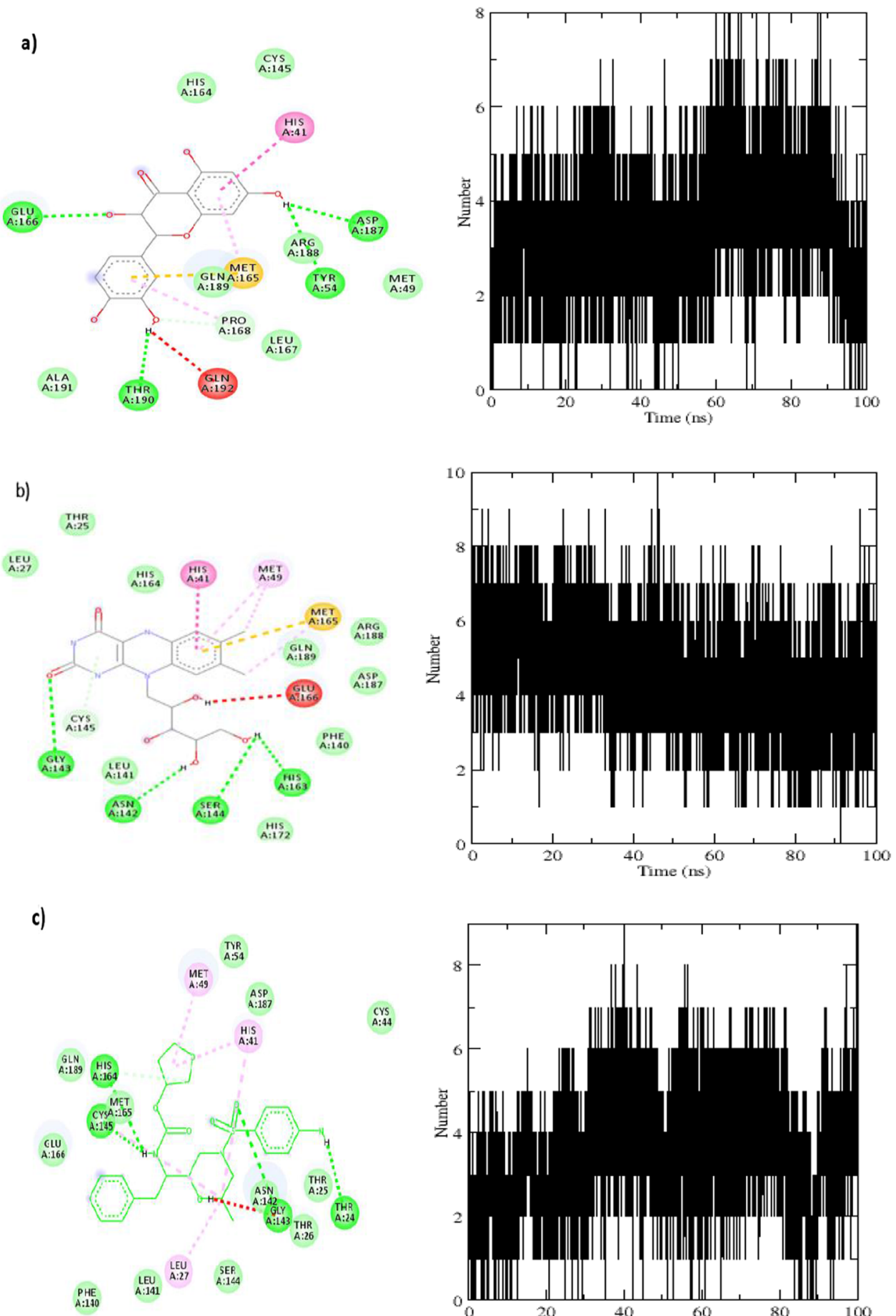

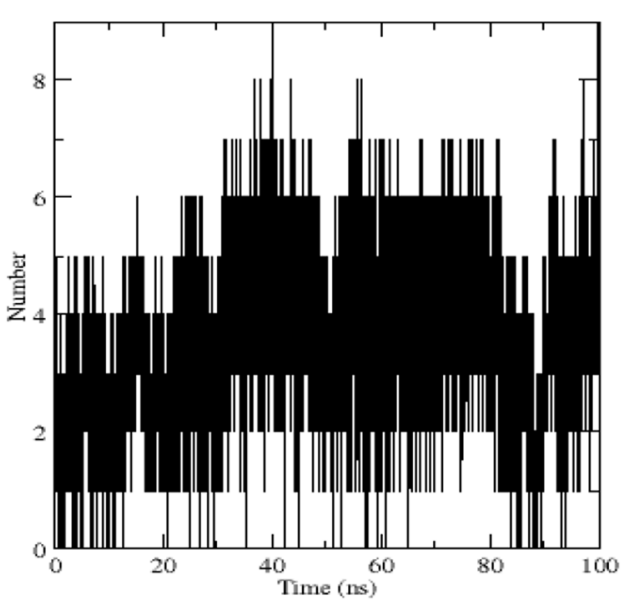

complex than the other inhibitors with the lowest $\mathrm{Rg}$ value. All other inhibitors have similar $\mathrm{Rg}$ as that of taxifolin and chloroquine (Fig. 4). Rg values for all protein-ligand are almost the same, meaning no change in the compactness and stability was maintained during MD simulation of all three $100 \mathrm{~ns}$ replica as shown in the Fig. (4 and S2).

SASA (solvent accessible surface area) values of all complexes are similar to taxifolin and chloroquine complex
(Fig. 5). The lower values of SASA specify the contracted nature of protein-ligand complex. We observed that the SASA plot has shown stability in docked complexes throughout simulation, which is significant for drug designing (Fig. 5 and S3).

The mobility of different residues of a protein in presence of drug was examined by measuring the RMSF values. RMSF results indicate that fluctuations were observed in 
Fig.2 (continued)
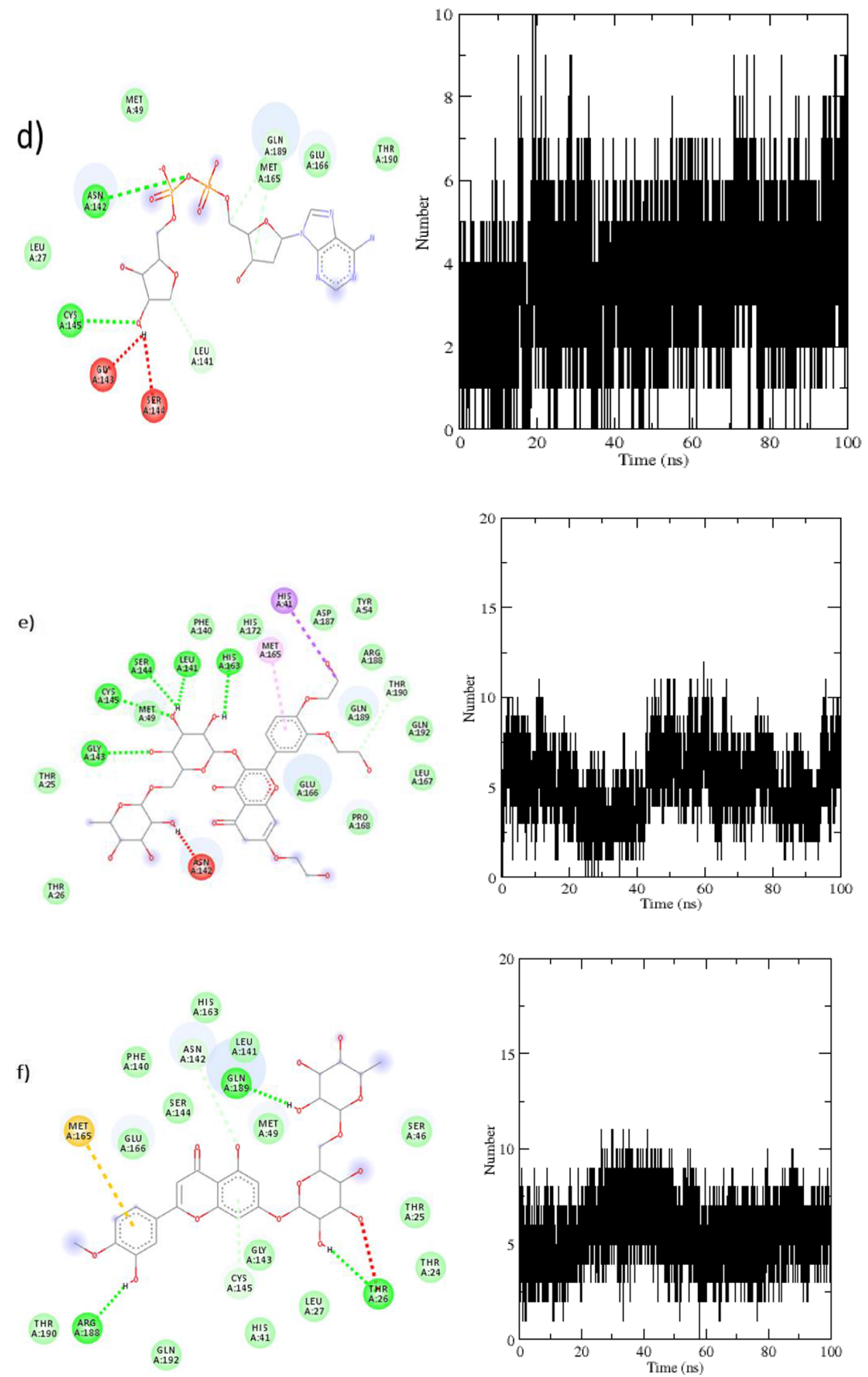

amprenavir, cefoperazone, diosmin, nadide, riboflavin and troxerutin compared with taxifolin and chloroquine. This showed that there are some conformational changes in protease to accommodate these inhibitors for creating a stable complex (Fig. 6).
The molecular dynamic simulation data supported the studies of molecular docking. It has provided important understanding of the interaction at the molecular level of all the inhibitor against main protease of COVID-19 that might be useful in drug development. 
Fig.2 (continued)

g)

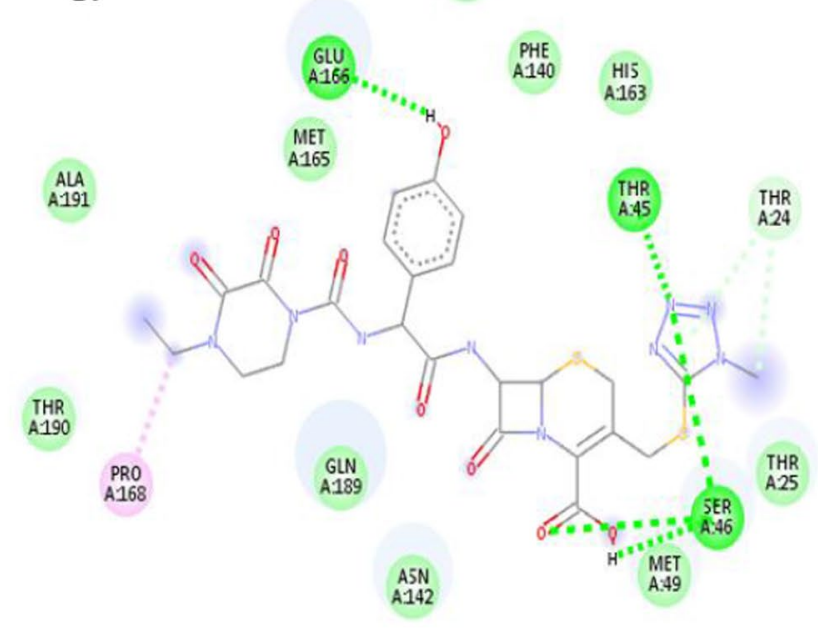

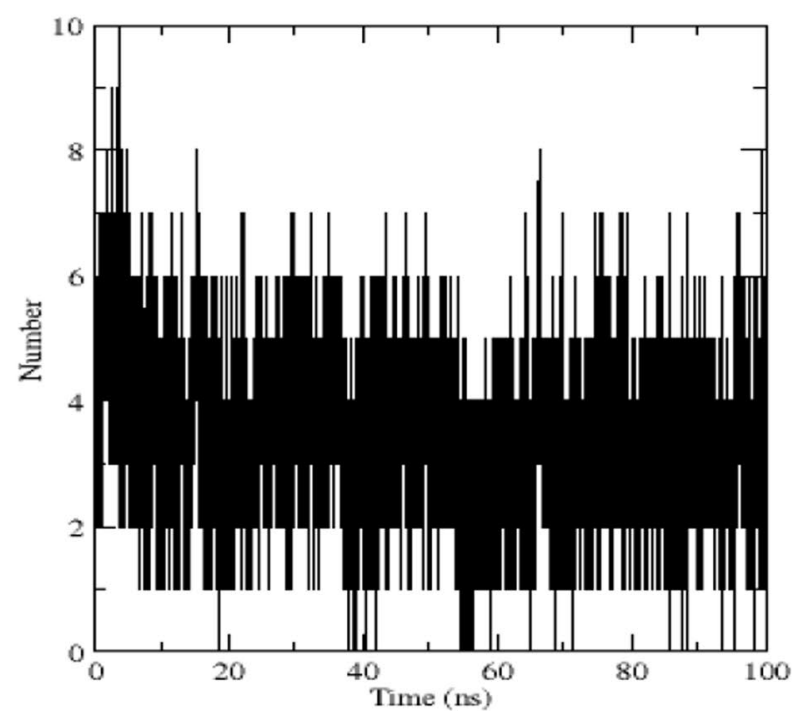

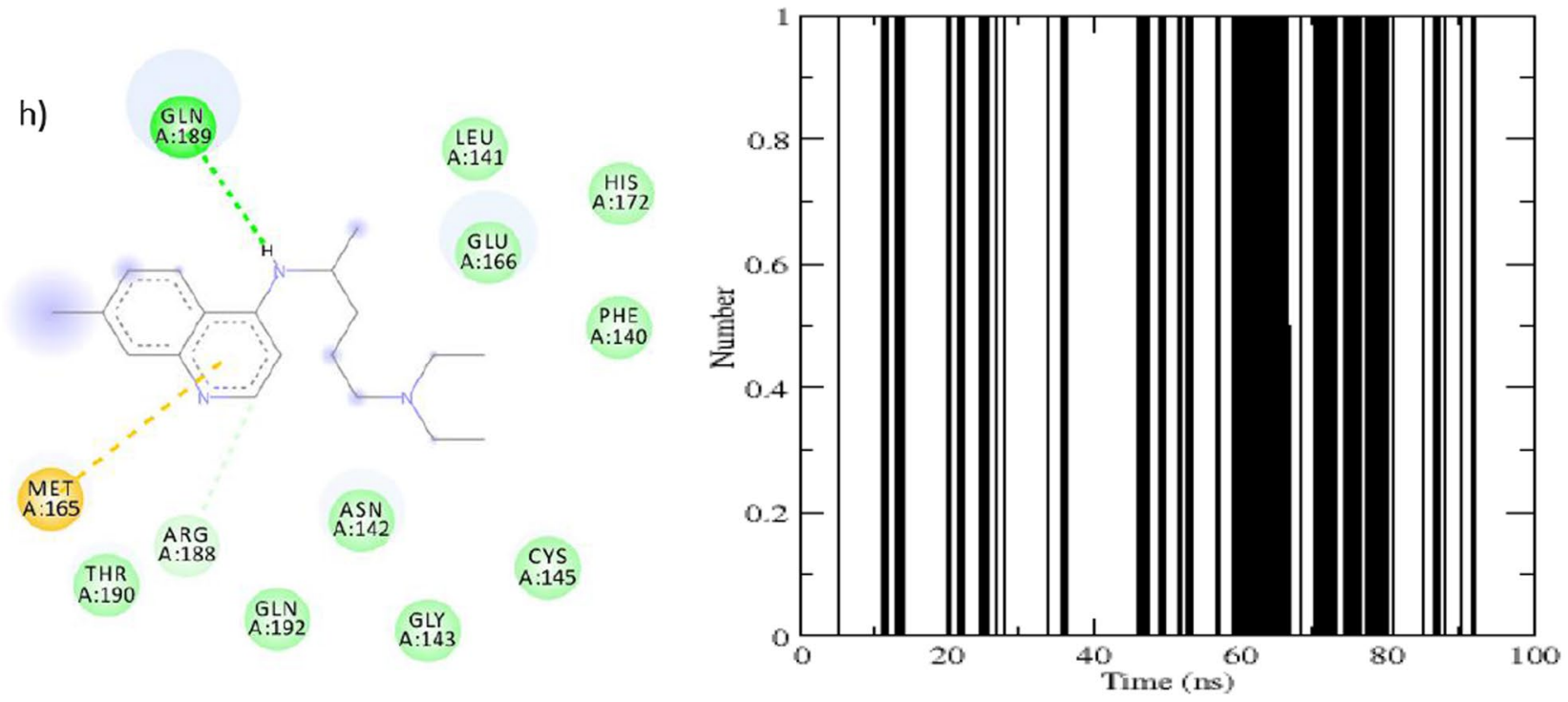

On the other hand, all the screened compounds show good binding energy (Table 1).

\section{Covalent docking}

Approximately $30 \%$ of the commercial drugs which target enzymes are covalent inhibitors [21]. The pharmacological studies reported benefits of covalent inhibitors that it can achieve longer drug residence times than non-covalent inhibitors [22] and has better target selectivity [23, 24]. RMSD lower than $2 \AA$ is generally considered as a principle for effective covalent docking [25].

Analysis of covalent interactions between inhibitors and main protease of coronavirus was done by using discovery studio software. There is a rule for effective covalent docking that the RMSD should be less than $2 \AA$ [25]. The RMSD value of diosmin-coronavirus main protease complex were found below $2.0 \AA$, while RMSD values of other inhibitors were found to be more than $2.0 \AA$ (Table 2 ). The results suggested that diosmin could form covalent bond with the Glu 166 of coronavirus main protease and may be considered as a good inhibitor (Fig. 7).

\section{Method}

\section{Preparation of protein}

The SARS-CoV-2 main protease structures (PDB ID:6LU7) was retrieved from RCSB Protein Data Bank (www.rcsb.org). The co-crystallized ligands and other heteroatoms were removed. All the water molecules 
Fig. 3 Backbone root mean square deviation (RMSD) values in nanometer (Y-axis) along with the time frame in nanosecond (X-axis)

Fig. 4 Radius of gyration of protease inhibitor complexes
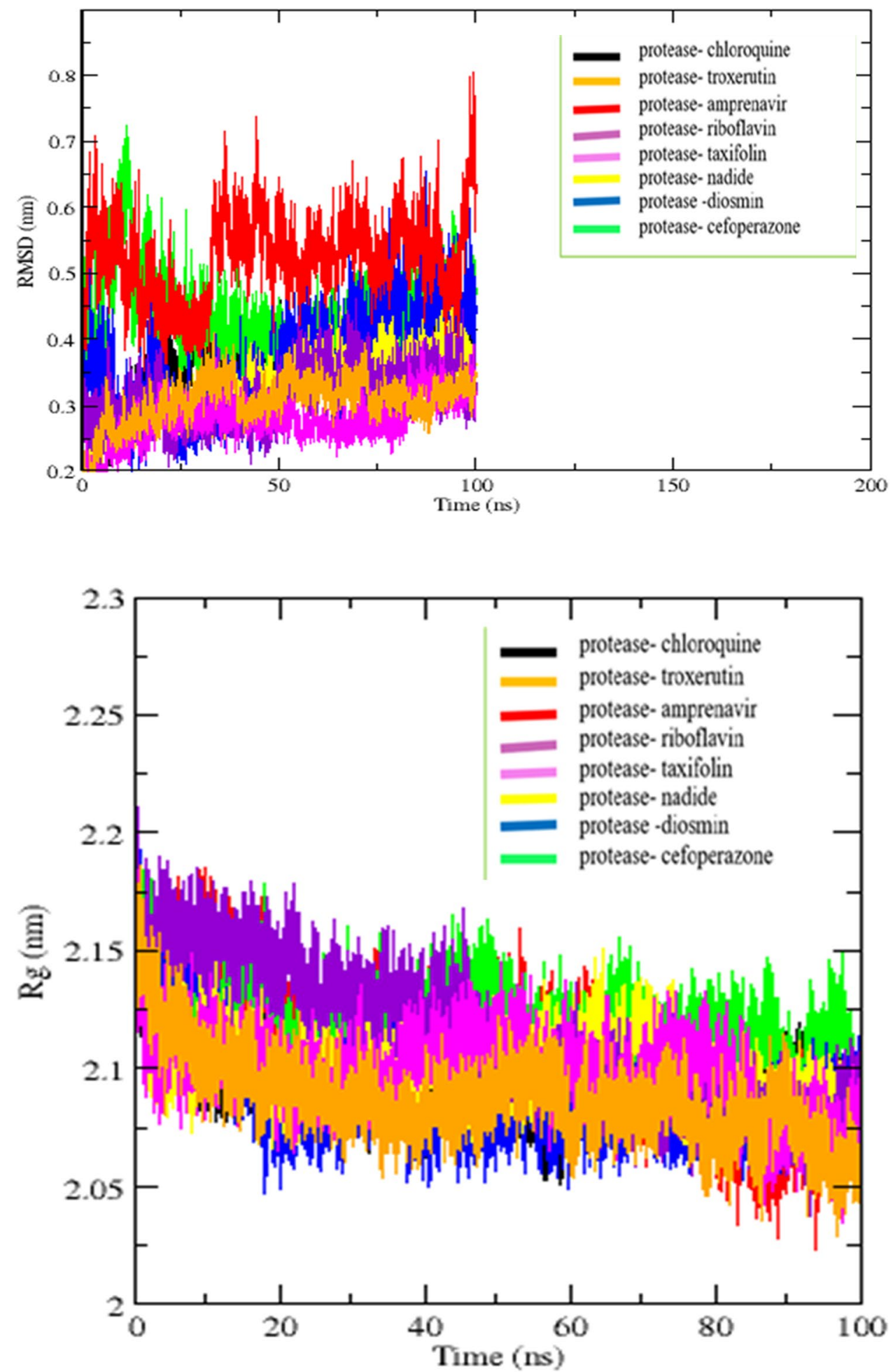

were removed from the original crystal structure before the protein preparation process; to analyse the structure and the bond order assigned, hydrogen atoms were added to the enzyme using Discovery Studio 2.5 package [26].

2. Preparation of ligand
Ligand database was downloaded from superdrug2 [27, 28] containing 6054 FDA-approved molecules. Hydrogen atoms were added in all the molecules. All the molecules were prepared by using Discovery Studio 2.5 package [26]. MM2 energy minimization tool of Chem3D17 7.1 software was used for minimization. 
Fig. 5 SASA values (Y-axis) along with the time frame (X-axis)

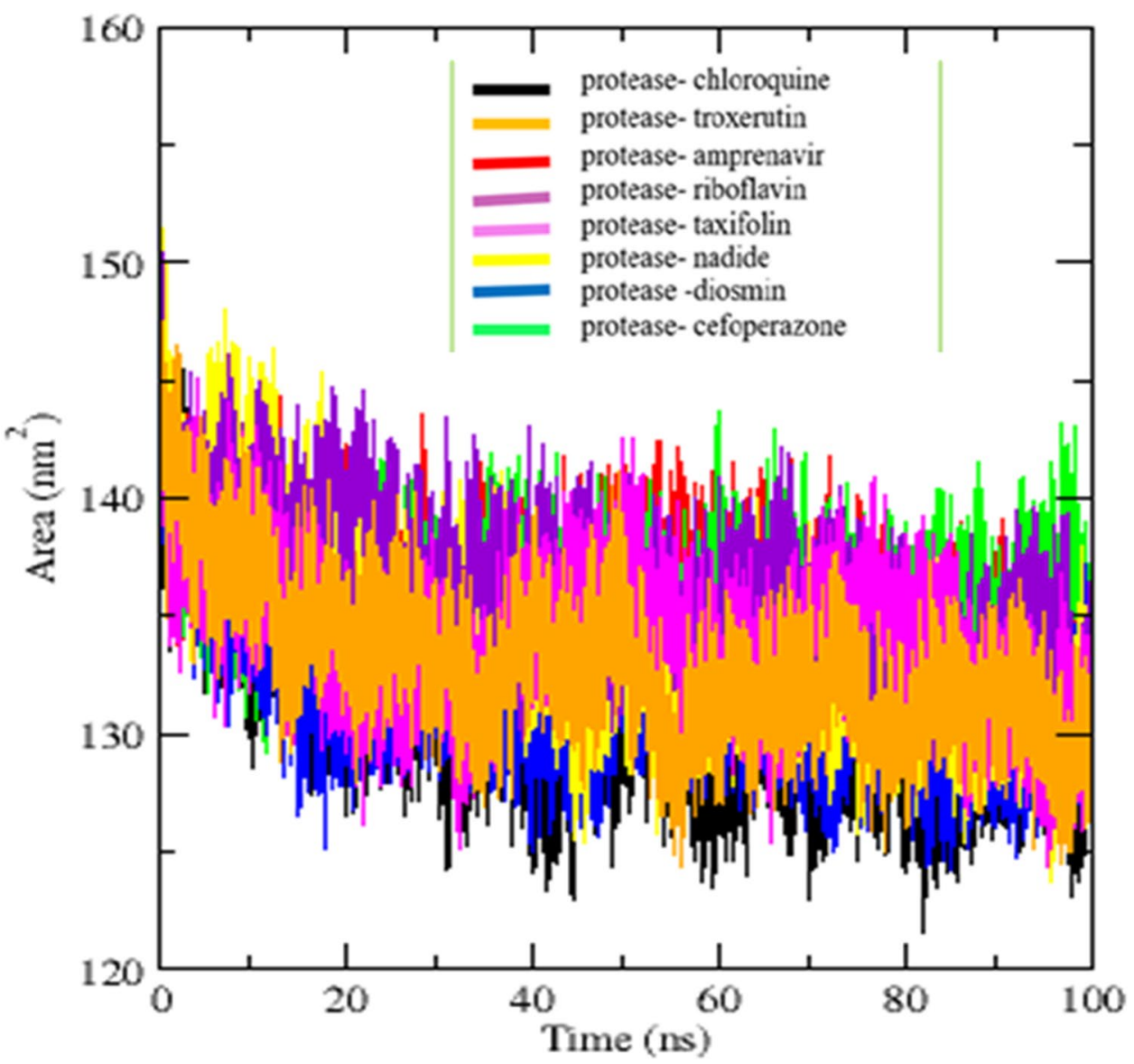

\section{Selection of positive control}

We have taken positive control to taxifolin (a natural compound) which is reported to have good in silico result against SARS-CoV-2 [29] and chloroquine, which is the main protease inhibitor of SARS-CoV-2[30].

4. High throughput virtual screening and molecular docking

High throughput virtual screening was performed by using GOLD (Genetic Optimization for Ligand Docking) 5.0 software [31]. Docking annealing parameters for van der Waals and hydrogen bonding were set to 5.0 and 2.5, respectively. The parameters used for genetic algorithm were considered as population size 100 , selection pressure 1.2, number of operations $1,00,000$, number of islands five, niche size 2 , migrate 10 , mutate 100 and crossover 100 . The selection of the compounds was based on the GOLD fitness score, favourable binding and molecular interactions with the active site residue. Further confirmation of the results was done by using AutoDock Vina software [32]. The framework for screening of the molecules was GOLD fitness score and binding energy from AutoDock Vina. Prior performing docking in AutoDock Vina, both receptors and ligands were prepared in AutoDock tools. Polar hydrogens were added to the receptor molecules, whereas non-polar hydrogens were merged. The binding site of the ligand was obtained from the available protein-ligand complex in PDB which was used as a reference to set the dimensions of grid box in all receptor molecules.

5. MD simulation and binding energy calculation

Molecular dynamics (MD) simulations for protein (one protomer)-ligand complex were performed by GROMACS (Groningen Machine for Chemical Simulation) 5.0 suite $[33,34]$ in combination with GROMOS96 $43 \mathrm{a} 1$ force field $[35,36]$. The ligand topologies were generated using the PRODRG webserver [37, 38]. Each docked complex was solvated with the extended simple point charge (SPC/E) water model in the cubic box. Each system was neutralized by adding counter ions which were followed by energy minimization. The equilibration steps, that is, NVT (isothermal-isochoric) then NPT (isothermal-isobaric) equilibration, were performed for 100 ps time [39]. Finally, three replicas of 100ns production run were carried out. Root mean square deviation (RMSD), root mean square fluctuation (RMSF), radius of gyration $(\mathrm{Rg})$, and hydrogen bond (H-bond) were calculated using gmx rms, gmx rmsf, gmx gyrate and gmx hbond modules of GROMACS utility. All 2D 


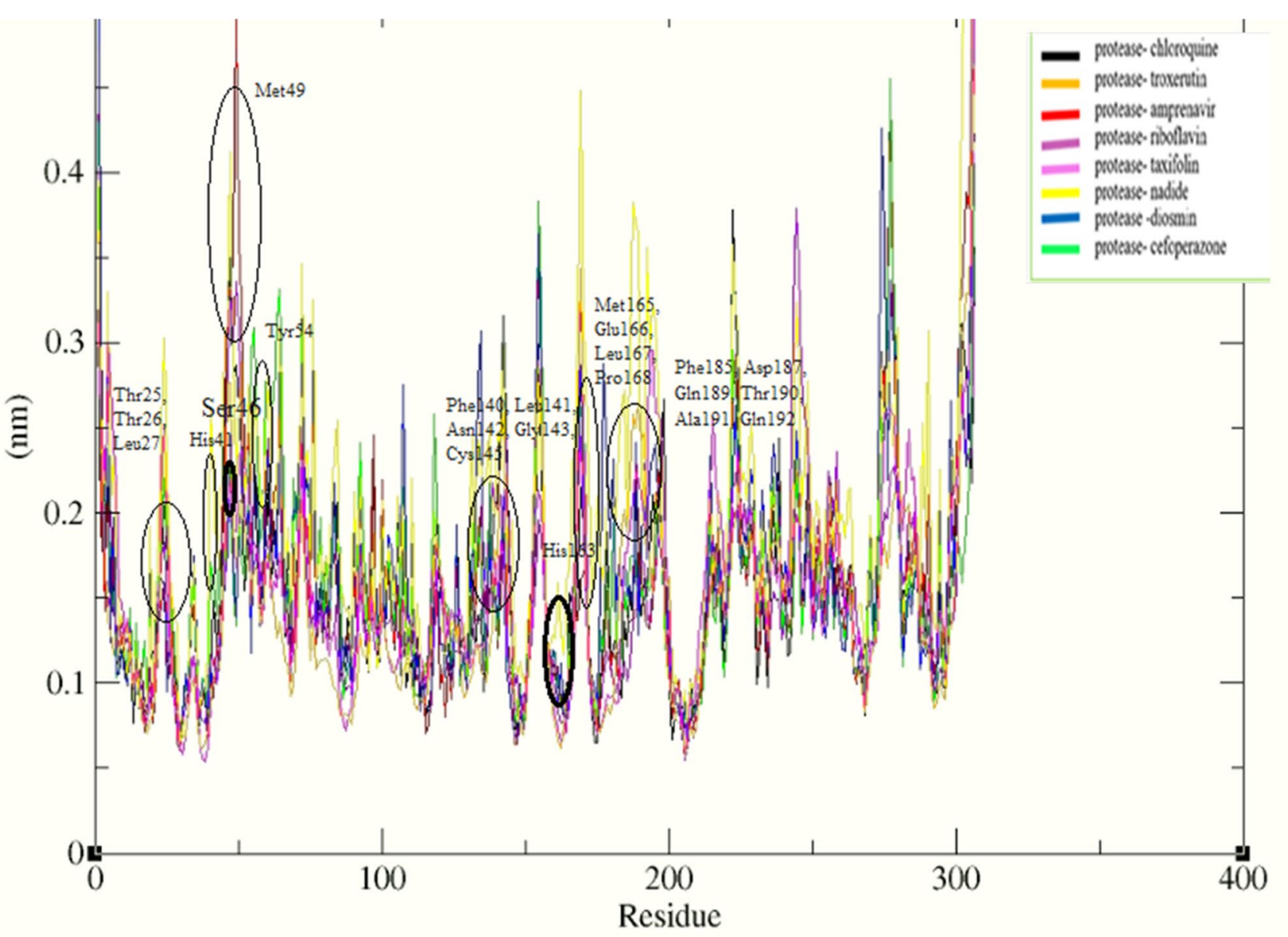

Fig. 6 RMSF plot of protease inhibitor complex with respect to amino acids residues

Table 2 RMSD value of covalent docking

\begin{tabular}{ll}
\hline Name of the complex & RMSD $(\AA)$ \\
\hline Diosmin & 1.96 \\
Troxerutin & 6.7 \\
Nadide & 3.2 \\
Riboflavin & 5.8 \\
Cefoperazone & 4.6 \\
Amprenavir & 4.0 \\
\hline
\end{tabular}

and 3D figures of proteins and ligands for visualisation were rendered in Discovery Studio Visualizer (Dassault Systems Biovia Corp).

Binding-free energy calculations for all the docked complex trajectories were performed by Molecular Mechanics/Poisson-Boltzmann Surface Area (MM/ PBSA) method of g_mmpbsa module [40, 41].

6. Covalent docking
Protein and ligand files were set up with the link atom identified and appear in both the protein and ligand input files. The complex PDB file and Mol2 file, which present receptor and ligand, respectively, were prepared with default parameters. Then, the link atom in the ligand was forced to attach to the link atom in the protein. The possible covalent interactions between the inhibitors and the protease were determined by performing covalent docking. It was done by Genetic Optimization for Ligand Docking (GOLD) 5.0 version [31]. RMSD value was calculated and result was analysed by the use of Discovery Studio 2.5 software [26].

\section{Conclusion}

Amprenavir, cefoperazone, riboflavin, diosmin, nadide and troxerutin approved for human therapeutic usage by FDA, found to act as COVID-19 main protease inhibitors, representing potential treatment option. All these drugs bind to the SARS-CoV-2 main protease conserved residues of 


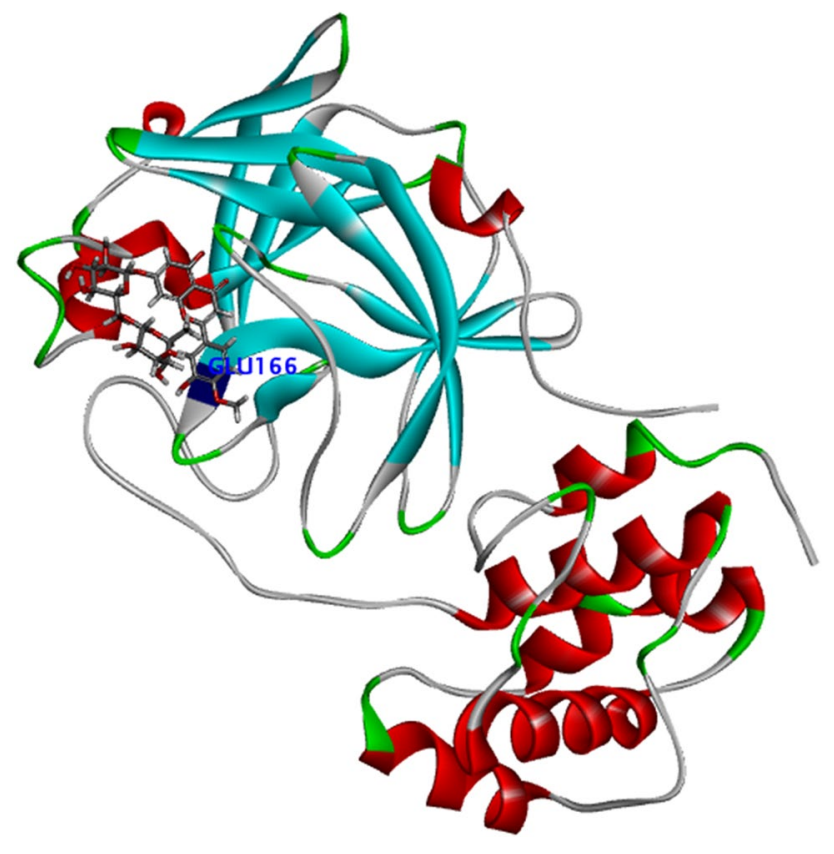

Fig. 7 Covalent interaction of diosmin with active site residue Glu 166 of SARS-CoV-2 main protease

substrate-binding pocket and form a remarkable number of non-covalent interactions. We have identified diosmin as an inhibitor which is binding covalently to the COVID-19 main protease, inhibiting the infection pathway of COVID-19.

In conclusion the predicted drugs can be used in current clinical trials to check effectiveness of molecules against COVID-19. The results of this study confirms initial reports however additional experiments are necessary to investigate the efficacy of these inhibitors by in vitro and in vivo studies followed by clinical trials against COVID-19 patients. But publishing these molecules in public domain may help community to fight with COVID-19 if any potential investigator proceeds for their trial. We recommend that these drug candidates be experimentally tested and used as a starting point for further design of a high-efficacy drug candidate.

Supplementary Information The online version contains supplementary material available at https://doi.org/10.1007/s00894-021-04923-w.

Acknowledgements We acknowledge the facility and support provided by the Department of Biotechnology, Ministry of science and technology, Government of India.

Authors' contributions NF performed study and wrote manuscript; AUK conceived the idea and guide and check the manuscript.

Funding Internal funds of the department supported the research.

Availability of data and material NA.

Code availability NA.

\section{Declarations}

Conflict of interest The authors declare no competing interests.

\section{References}

1. Chen N, Zhou M, Dong X et al (2020) Epidemiological and clinical characteristics of 99 cases of 2019 novel coronavirus pneumonia in Wuhan, China: a descriptive study. Lancet 395(10223):507-513. https://doi.org/10.1016/S0140-6736(20) 30211-7

2. World Health Organisation, International Health Regulations Emergency Committee on novel coronavirus in China. https:// https://www.who.int/health-topics/coronavirus/origins-of-thevirus 30 march 2021

3. Stoermer, M. J. (2020) Homology Models of the Papain-Like Protease PLpro from Coronavirus 2019-nCoV. ChemRxiv. Preprint. https://doi.org/10.26434/chemrxiv.11799705.v1

4. Zhou P, Yang X, Wang X et al (2020) A pneumonia outbreak associated with a new coronavirus of probable bat origin. Nature 579:270-273. https://doi.org/10.1038/s41586-020-2012-7

5. Yang S, Chen SJ, Hsu MF et al (2006) Synthesis, crystal structure, structure-activity relationships, and antiviral activity of a potent SARS coronavirus 3CL protease inhibitor. J Med Chem 49(16):4971-4980. https://doi.org/10.1021/jm0603926

6. Ghosh AK, Osswald HL, Prato G (2016) Recent Progress in the Development of HIV-1 Protease Inhibitors for the Treatment of HIV/AIDS. J Med Chem 59(11):5172-5208. https://doi.org/10. 1021/acs.jmedchem.5b01697

7. Zhijian Xu, Peng Cheng, Shi Yulong et al (2020) Nelfinavir was predicted to be a potential inhibitor of 2019-nCov main protease by an integrative approach combining homology modelling, molecular docking and binding free energy calculation. bioRxiv 1201:1-20. https://doi.org/10.1101/2020.01.27.921627

8. Liu X, Wang XJ (2020) Potential inhibitors against 2019-nCoV coronavirus $\mathrm{M}$ protease from clinically approved medicines. $\mathbf{J}$ Genet Genomics 47(2):119-121. https://doi.org/10.1016/j.jgg. 2020.02.001

9. Lai MMC, Holmes KV (2001) Coronaviridae: the viruses and their replication. In: Knipe DM, Howley PM (eds) Fields virology. Lippincott Williams \& Wilkins, Philadelphia, pp 1163-1179

10. Jin Z, Du X, Xu Y et al (2020) Structure of $M^{\text {pro }}$ from SARSCoV-2 and discovery of its inhibitors. Nature 582(7811):289293. https://doi.org/10.1038/s41586-020-2223-y

11. Li Y, Zhang J, Wang $\mathrm{N}$ et al (2020) Therapeutic drugs targeting 2019-nCoV main protease by high-throughput screening. bioRxiv, 2020.01.28.922922

12. Zhang H, Saravanan KM, Yang Y et al (2020) Deep learning based drug screening for novel coronavirus 2019-nCov. Preprints.19, 1-17.2020020061. https://doi.org/10.20944/prepr ints202002. 0061.v1

13. Pillaiyar T, Manickam M, Namasivayam V et al (2016) An overview of severe acute respiratory syndrome-coronavirus (SARSCoV) 3CL protease inhibitors: Peptidomimetics and small molecule chemotherapy. J Med Chem 59:6595-6628. https://doi. org/10.1021/acs.jmedchem.5b0146

14. Raugi DN, Smith RA, Gottlieb GS (2016) Four Amino Acid Changes in HIV-2 Protease Confer Class-Wide Sensitivity to Protease Inhibitors. J Virol 90:1062-1069. https://doi.org/10. 1128/JVI.01772-15

15. Zhang L, Lin D, Sun X et al (2020) Crystal structure of SARS$\mathrm{CoV}-2$ main protease provides a basis for design of improved 
$\alpha$-ketoamide inhibitors. Science 368(6489):409-412. https://doi. org/10.1126/science.abb3405

16. Dai W, Zhang B, Jiang XM, Su H, Li J, Zhao Y, Xie X, Jin Z, Peng J, Liu F, Li C, Li Y, Bai F, Wang H, Cheng X, Cen X, Hu S, Yang X, Wang J, Liu X, ... Liu H (2020) Structure-based design of antiviral drug candidates targeting the SARS-CoV-2 main protease. Science (New York, N.Y.) 368(6497): 13311335. https://doi.org/10.1126/science.abb4489

17. Fischer A, Sellner M, Neranjan S et al (2020) Potential Inhibitors for Novel Coronavirus Protease Identified by Virtual Screening of 606 Million Compounds. Int J Mol Sci 21(10):3626. https://doi. org/10.3390/ijms21103626

18. Danishuddin M, Khan A, Faheem M et al (2014) Structure-based screening of inhibitors against KPC-2: designing potential drug candidates against multidrug-resistant bacteria. J Biomol Struct Dyn 32(5):741-750. https://doi.org/10.1080/07391102.2013. 789988

19 Nagpal I, Raj I, Subbarao N et al (2012) Virtual screening, identification and in vitro testing of novel inhibitors of O-acetyl-Lserine sulfhydrylase of Entamoeba histolytica. PLoS One 7:e30305. https://doi.org/10.1371/journal.pone.0030305

20. Wade RC, Goodford PJ (1989) The role of hydrogen-bonds in drug binding. Prog Clin Biol Res 289:433-444

21. Singh J, Petter RC, Baillie TA et al (2011) The resurgence of covalent drugs. Nat Rev Drug Discov 10:307-317. https://doi. org $/ 10.1038 / \mathrm{nrd} 3410$

22. Bradshaw JM, McFarland JM, Paavilainen VO et al (2015) Prolonged and tunable residence time using reversible covalent kinase inhibitors. Nat Chem Biol 11:525-531. https://doi.org/10.1038/ nchembio. 1817

23. Hansen R, Peters U, Babbar A et al (2018) The reactivity-driven biochemical mechanism of covalent KRAS(G12C) inhibitors. Nat Struct Mol Biol. https://doi.org/10.1038/s41594-018-0061-5

24. Goedken ER, Argiriadi MA, Banach DL et al (2015) Tricyclic covalent inhibitors selectively target Jak3 through an active site thiol. J Biol Chem 290:4573-4589. https://doi.org/10.1074/jbc. M114.595181

25. De Cesco S, Kurian J, Dufresne C et al (2017) Covalent inhibitors design and discovery. Eur J Med Chem 138:96-114

26. BIOvIA DS (2015) Discovery studio modeling environment; Dassault Systemes, Release: San Diego, 2015; Vol. 4

27. Siramshetty VB, Eckert OA, Gohlke B-O et al (2018) SuperDRUG2: a one stop resource for approved/marketed drugs. Nucleic Acids Res 46(D1):D1137-D1143. https://doi.org/10. 1093/nar/gkx1088

28. Goede A, Dunkel M, Mester N et al (2005) SuperDrug: a conformational drug database. Bioinformatics 21(9):1751-1753. https:// doi.org/10.1093/bioinformatics/bti295

29. Fischer A, Sellner M, Neranjan S et al (2020) Potential Inhibitors for Novel Coronavirus Protease Identified by Virtual Screening of 606 Million Compounds. Int J Mol Sci 21(10):3626. https://doi. org/10.3390/ijms21103626

30. Wang M, Cao R, Zhang L et al (2020) Remdesivir and chloroquine effectively inhibit the recently emerged novel coronavirus
(2019-nCoV) in vitro. Cell Res 30(3):269-271. https://doi.org/10. 1038/s41422-020-0282-0

31. Jones G, Willett P, Glen RC (1995) Molecular recognition of receptor sites using a genetic algorithm with a description of desolvation. J Mol Biol 245(1):43-53. https://doi.org/10.1016/ s0022-2836(95)80037-9

32. Trott O, Olson AJ (2010) AutoDock Vina: improving the speed and accuracy of docking with a new scoring function, efficient optimization, and multithreading. J Comput Chem 31(2):455-461. https://doi.org/10.1002/jcc. 21334

33. Singh A, Somvanshi P, Grover A (2019) Drug repurposing against arabinosyl transferase (EmbC) of Mycobacterium tuberculosis: Essential dynamics and free energy minima based binding mechanics analysis. Gene 693:114-126. https://doi.org/10.1016/j. gene.2019.01.029

34. Singh A, Somvanshi P, Grover A (2019) Pyrazinamide drug resistance in RpsA mutant $(\Delta 438 \mathrm{~A})$ of Mycobacterium tuberculosis: Dynamics of essential motions and free-energy landscape analysis. J Cell Biochem 120:7386-7402. https://doi.org/10.1002/jcb. 28013

35. Singh A, Grover S, Sinha S et al (2017) Mechanistic principles behind molecular mechanism of rifampicin resistance in mutant RNA polymerase beta subunit of mycobacterium tuberculosis. J Cell Biochem 118(12):4594-4606. https://doi.org/10.1002/jcb. 26124

36. Van Gunsteren WF, Billeter SR, Eising et al (1996) Biomolecular simulation: the GROMOS96 manual and user guide; Vdf Hochschulverlag AG an der ETH Zürich. 1996; Vol. 86

37. Schüttelkopf AW, van Aalten DM (2004) PRODRG: a tool for high-throughput crystallography of protein-ligand complexes. Acta Crystallogr D Biol Crystallogr 60(Pt 8):1355-1363. https:// doi.org/10.1107/S0907444904011679

38. Sinha S, Tyagi C, Goyal S et al (2016) Fragment based G-QSAR and molecular dynamics based mechanistic simulations into hydroxamic-based HDAC inhibitors against spinocerebellar ataxia. J Biomol Struct Dyn 34(10):2281-2295. https://doi.org/ 10.1080/07391102.2015.1113386

39. Sinha S, Verma S, Singh A et al (2018) Simulation based investigation of deleterious nsSNPs in ATXN2 gene and its structural consequence toward Spinocerebellar Ataxia. J Cell Biochem 119(1):499-510. https://doi.org/10.1002/jcb.26209

40. Kumari R, Kumar R, Lynn A (2014) G mmpbsa A GROMACS Tool for High-Troughput MM-PBSA Calculations. J Chem Inf Model 54(7):1951-62

41. Kumari R, Lynn AM (2011) Application of MM/PBSA in the prediction of relative binding free energy: Re-scoring of docking hit-list. J Nat Sci Biol Med 2(3):92

Publisher's note Springer Nature remains neutral with regard to jurisdictional claims in published maps and institutional affiliations. 